\title{
Segmentation on the Dental Periapical X-Ray Images for Osteoporosis Screening
}

\author{
Enny Itje Sela \\ Department of Informatics \\ STMIK AKAKOM \\ Yogyakarta, Indonesia
}

\author{
Sri Hartati ${ }^{1}$, Agus Harjoko ${ }^{1}$, Retantyo Wardoyo ${ }^{1}$, \\ Munakhir MS ${ }^{2}$ \\ ${ }^{1}$ Department of Computer Science and Elecronics \\ ${ }^{2}$ Department of DentomaxillofacialRadiology \\ UniversitasGadjahMada \\ Yogyakarta, Indonesia
}

\begin{abstract}
Segmentation on the trabecular of dental periapicalX-Ray images is very important for osteoporosis screening. Existing methods do not perform well in segmenting the trabecular of dental periapical in X-Ray images due to the presence of large amount of spurious edges. This paper presents a combination of tophat-bothat filtering, histogram equalization contrasting and local adaptive thresholding approach for automatic segmentation of dental periapical in $\mathrm{X}$-Ray images. The qualitative evaluation is done by a dentist and shows that the proposed segmentation algorithm performed well the porous of trabecular features of dental periapical. The quantitative evaluation used fuzzy classification based on neural network to classify these features. It were found accuracy rate to be $99,96 \%$ for training set and around $65 \%$ for testing set for a dataset of 60 subjects.
\end{abstract}

Keywords-dental periapical X-Ray; osteoporosis; porous trabeculae; segmentation.

\section{INTRODUCTION}

Computer Aided Diagnosis (CAD) has been showing greater significance for bringing such effective and voluminous number of medical images possible [1]. Computer algorithms play a major part in extracting data from a medical image such as facilitating and automating the delineation of anatomical structures, identifying bone cracks and various other biomedical applications [2]. One major of such techniques lie in the realm of X-Ray image analysis.

Radiograph is the oldest form of non-invasive, painless, economical and easily observable digital imaging technique and it is widely used during various stages of treatment. Bone structure analysis is a technique that requires details that can only be obtained as features from an X-Ray image. A method used to extract such information is called segmentation which involves the grouping or classification of pixels in an image into groups containing common characteristics [2].

Segmentation of bones in medical images is very important for medical applications such as fracture detection. Segmentation of bones in X-ray images is a very difficult and challenging task that is not well understood [3]. In fact, the segmentation alone can determine the eventual success or failure of the analysis at hand. Segmentation involves working on a number of images processing tasks such as noise removal and image enhancement. In an anterior-posterior periapicalXRay, the image is too noisy, too blur and too dark. As a consequence, some boundary edges of trabecular and its porous may appear to be connected each other. These difficulties cause general segmentation methods inappropriate.

Combination of the tophat filtering, contrast stretching, and Otsu thresholding methods [16] was fail to segment our periapicalX-Ray images. Other methods such as combination of the tophat filtering, contrast stretching, wiener noise removal, color quantization and spatial segmentation [4], combination of a $3 \times 3$ median filtering, binarization, and erosion [5], combination of the Gausian filtering, subtraction the original image with the image of the filtering, then Otsu threshold method [6] were tried to segment periapical X-ray images and result unsuccessfully segmentation.

Another method has been proposed to perform for segmentingperiapical X-ray images. The segmented image consists of the trabecular and it's porous. The porous then extracted as features of osteoporosis disease. These features can be used to predict a subject's femoral/lumbar World Health Organization (WHO) bone mineral density. The WHO classifiers the subjects as follows: osteoporotic, osteopenia, and normal [7].

\section{MATERIALS}

\section{A. Subjects}

Ethical clearance has been obtained from the local ethics committee of Faculty of Dentistry, UniversitasGadjahMada, Yogyakarta for this study. The informed consent obtained from all subjects. The subjects consisted of 60 consenting females (average age 61.3 years;and ranges 41-80). Each subject was collectedsubject's age, height, weight and BMI at the time of radiographic examination. None of the subjects was known to have endocrine, metabolic, or skeletal disorder. None of the subjects was on hormonal replacement therapy or taking calcitonin, bisphosphonates, or fluorides except of low doses of calcium or vitamin D.

\section{B. Dental Periapical X-Ray}

All periapicalX-Ray carried out from the Department of Radiology of Prof. SoedomoDental Hospital, Faculty of Dentistry UniversitasGadjahMada (Fig 1). They were taken by a radiographer using dental X-ray Villa SISTEMI medicaliendos ACP CEI specification $70 \mathrm{kVp}, 8 \mathrm{~mA}$, and $3.2 \mathrm{~s}$ and the image receptor photostimulable phosphor plate (PSP). 
Periapical X-Ray processing used digital radiography (DBSWin 4.5, Durr Dental). The size of this bitmap image is 1252 x 1645 pixels.

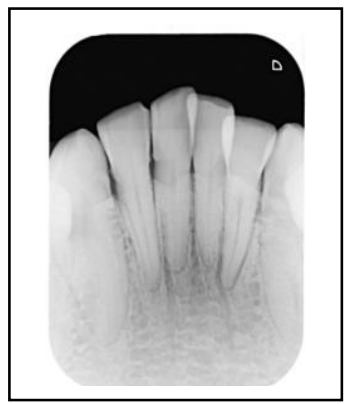

Fig.1. Dental Periapical X-Ray Image

\section{C. $B M D$}

Assessment of Bone Mineral Density(BMD) on femoral neck and lumbar spine carried out from the Department of Radiology Dr. Sardjito Hospital using densitometer Dual energy X-ray absorptiometry (DXA) specifications $76 \mathrm{kV}$,

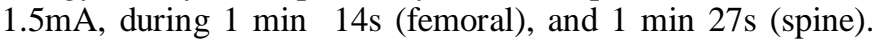
The procedure is operated by a radiographer. Subjects were classified into one of three groups contained women who were classified according to the WHO classification.

\section{Methodology AND DESIGN}

\section{A. Methodology}

In this study, there are some steps to obtain the segmented images. First step is selection ROIs from originally images. Second step is segmentation process. Last step is evaluation process. All of the steps can be shown on Figure 2.

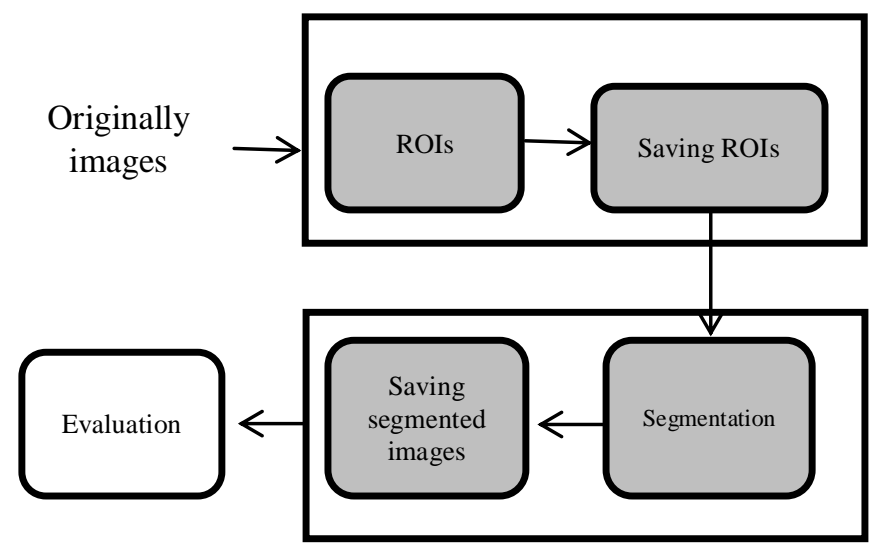

Fig.2. Research Methodology

\section{Selection of ROIs.}

All of ROIswere selected around the trabeculararea for each patient, as decided independently by an observer. To obtain the ROIs, a dentist should make a point on the trabecular area. Then the system makes rectangle automatically. The maximum size of all rectangles was 400 x300 pixels, with a pixel size of $0.02 \mathrm{~mm}$. All of ROIs are saved in bitmap format.

\section{Segmentation.}

The segmentation process is used to separate the trabecular and porous objects all ROIs resulted from previous step. Trabecular are presented in the white pixel and porous presented in black pixel. Data X-Ray were scanned at $600 \mathrm{dpi}$, made uniform in overall intensity by blurring the image by applying a tophat bothat filtering. Top hat filtering and bottom hat filtering can be used together to enhance contrast in an image. Contrasting by histogram equalization[8]was used to distribute the intensity of the pixels in the interval [ $\left[\begin{array}{ll}0 & \text {.. 255], }\end{array}\right.$ and last, adaptive threshold $[10,11]$ with a window of $40 \times 40$ pixels. Thisprocess was resulted a binary image.

Segmentation procedureis as follows [8] :

Input: bitmap format images, gray scale, 8 bit (img) Output: a binary image (outimage)

\section{1) Filtering}

Tophat performs morphological top-hat filtering on the grayscale or binary input image using the structuring element. Steps of tophat filtering are:

a. Erosion.

$$
\begin{aligned}
& I_{2}=I_{1} \Theta B \ldots \ldots \ldots \ldots .(1) \\
& \mathrm{I}_{1}=\text { initial image, } \mathrm{I}_{2}=\text { image erosion } \\
& \mathrm{B}=\text { structure element disk }(\mathrm{r}=1)
\end{aligned}
$$

Steps to perform erosion are:

a) Comparing each pixel with the color of the pixel center $I_{1} B$ by superimposing $B$ with $I_{1}$ so that the center of $B$ is exactly same with the image pixel positions $I_{1}$.

b) If all the pixels in $B$ exactly the same as all the pixels in $I_{1}$ (foreground) pixel $I_{1}$ then set its value to the foreground pixel value, otherwise the value of the pixel value will be set according to the value of the background.

b. Dilation on I2.

$$
\begin{gathered}
I_{3}=I_{2} \oplus B \ldots \ldots . . . . \\
I_{3}=\text { dilation result. }
\end{gathered}
$$

Steps to perform dilation are

c) Comparing each pixel with the color of the pixel center I2 B by superimposing B with 12 so that the center of B is exactly same with the image pixel positions $I 2$.

d) If at least one pixel to pixel B is equal to the value of the object (foreground) in I2 then the pixel value is set to the foreground pixel value and if all the pixels that are related to the background pixel value set in I2 are like background pixel value.

$$
\text { c. Subtract. }
$$

$$
\mathrm{I}_{4}=\mathrm{I}_{1}-\mathrm{I}_{3}
$$

Bothat performs morphological bottom-hat filtering on the grayscale or binary input image. This process is the reverse of the tophat. The steps for this process are: a. Dilation on $\mathrm{I}_{1}$

$$
I_{5}=I_{1} \oplus B
$$


b. The process of erosion on I5

$I_{6}=I_{5} \Theta B$

c. Subtract $\mathrm{I}_{1}$ to $\mathrm{I}_{6}$

$I_{7}=I_{1}-I_{6}$

1. Contrasting.

This step is used to distribute the intensity of the pixels in the interval [0..255]. The equation to perform histogram equalization can be seen below.

$n(g)=\max \left(0\right.$, round $\left[\left(L-1 * \frac{c(g)}{N}\right]-1\right)$

$\mathrm{N}=$ number of pixels in the image vectors

$\mathrm{g}=$ initial value of gray level value of (L-1)

$\mathrm{L}=$ maximum value of gray level

$c(g)=$ number of pixels that have a value equal to $\mathrm{g}$ or less

$$
c(g)=\sum_{i=1}^{g} h(i)
$$

$\mathrm{g}=1,2$,

$h(i)=$ initial histogram.

2. Adaptive segmentation thresholding with the average value[9][10]

a. Set a constant value of $\mathrm{N}=40$

b. Make a subimage window of $\mathrm{K} 1$ with size

$\mathrm{NxN}$ on the image.

c. Find the average pixel intensity subimage K1

d. Perform the threshold with the average value

of $\mathrm{K} 1$

if $\mathrm{K} 1(\mathrm{i}, \mathrm{j})<=$ threshold

$$
\text { outimage }(i, j)=1 \text {; }
$$

else

$$
\text { outimage }(i, j)=0
$$

\section{RESUlT}

A custom computer program was used to obtain morphologic variables from the digitized periapical X-Ray. The same ROIs used for the radiographic jaw density were used for this analysis. A dentist used our system with give a point on the trabecular using mouse (Figure 3). Then, the system made $400 \times 300$ pixel of ROI automatically.Figure 4 shows the result of ROI from our periapical dental X-Ray.

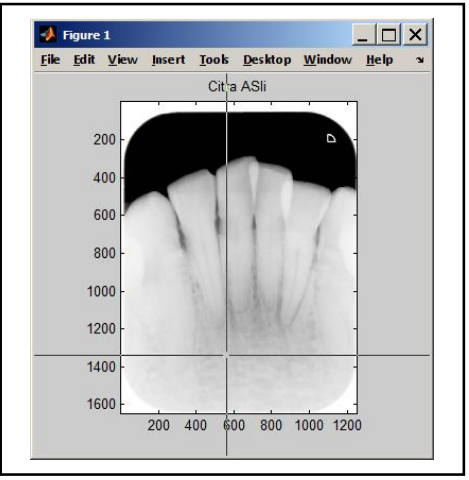

Fig.3. Input a point on the trabecular bone

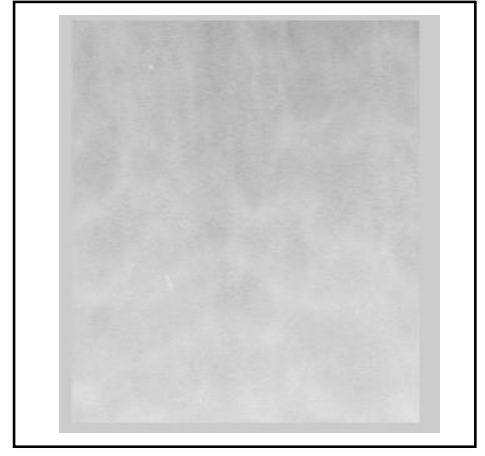

Fig.4. ROI of trabecular bone from digitized X-Ray

Based on the above segmentation procedure, the results can be presented as follows. Fig 5 shows the filtering image using tophat and bothat filtering of ROI. This image uses tophat and bothat filtering with a disk-shaped structuring element disk $(r=1)$ to remove the uneven background illumination from an image and enhance contrast in the image.

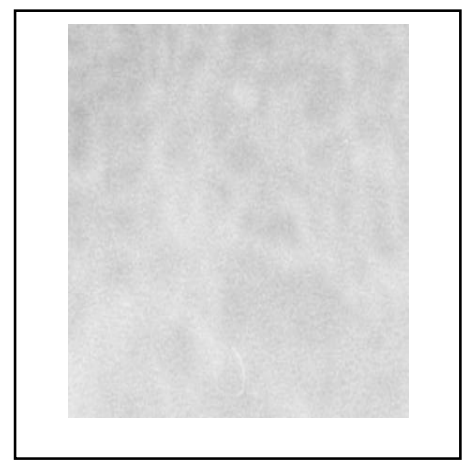

Fig.5. Result of tophat and bothat filtering of ROI

Fig 6 shows the result of adjusting image seen in Fig 5 using histogram equalization. This image can show the trabeculae (white pattern) and porous (black pattern) clearly.

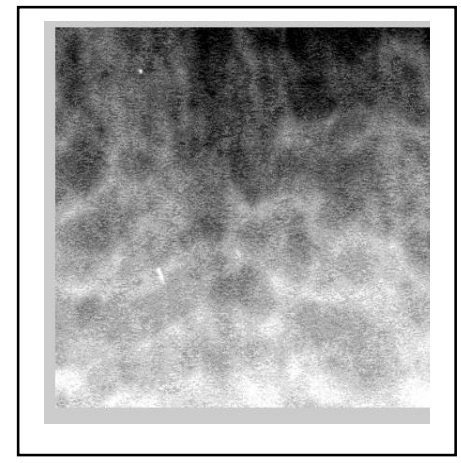

Fig.6. Contrasting image seen in Fig 5

Fig 7 shows the result of adaptive local threshold on adjusted image seen in Fig 6 . The size of the resulting binary image is smaller than the original image depending on the size of the window. In this study, the size of the window is $40 \times 40$ 
pixels. This operation result a binary image with $360 \times 260$ pixels in size.

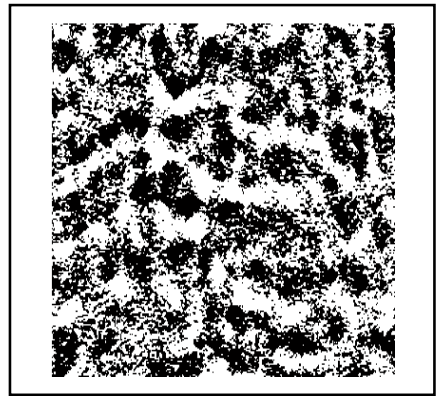

Fig.7. Segmented Image

There is a difficulty to evaluate the segmentation. A dentist then evaluate segmented image by comparing the segmented image with another segmented image resulted in previous research [6][15]. It is said that the binary image resulted from the segmentation is well. In this study, the quantitated evaluation is performed for classification. The binary image is analyzed to measure features including porosity[13],number of vertex of porous [11] and perimeter of porous [11] after morphology operation (clear border, filling, dilation, and erosion). The features are saved in text data format. Then thefuzzypattern

classificationusingbackpropagationlearningalgorithm [14] is used to training and testing these features.

Samples of 60 acquired dataset are divided into training set (12 normal, 11 osteopenia, 7 osteoporotic) to generate weight of neural network and testing set (14 normal, 12 osteopenia, 4 osteoporotic) to test the capability of system to get the output class.The result of classification using multilayer backpropagation with $10^{-6} \mathrm{MSE}$ (MSE goal was $6.90 \times 10^{-7}$ ), 650 epochs, 0.1 learning rate, and sigmoidal transfer function. The performance of neural network is shown at Fig8.

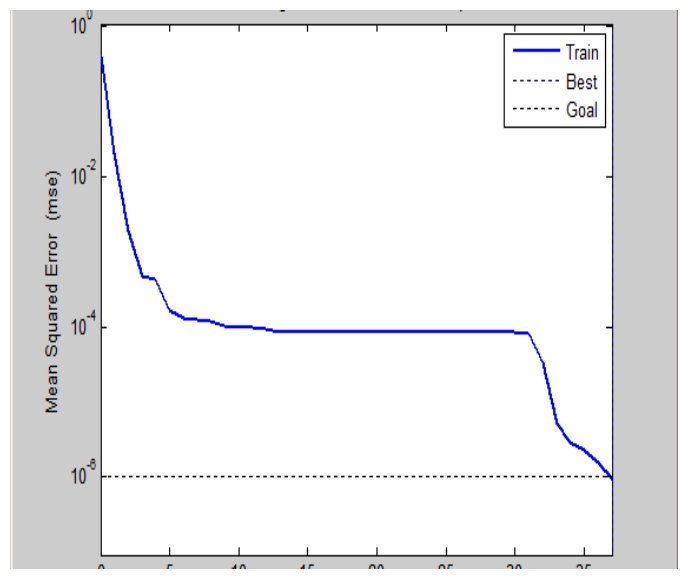

Fig.8. Performance Neural Network

The training results were found accuracy rate to be $99,96 \%$ and are shown as Table 1 . Test results were found to be around $65 \%$ and shown in Table 2 .
TABLE I. Percentage ACCURACY TRAINING RESUlT

\begin{tabular}{|l|l|l|}
\hline \multicolumn{1}{|c|}{ Class } & \multicolumn{1}{c|}{ Femur } & Lumbar \\
\hline Normal & 99,96 & 99,83 \\
\hline Osteopenia & 99,83 & 99,93 \\
\hline Osteoporotic & 99,99 & 99,96 \\
\hline
\end{tabular}

TABLE II. PERCENTAGes ACCURACY TESTING

\begin{tabular}{|l|l|l|}
\hline \multicolumn{1}{|c|}{ Class } & \multicolumn{1}{c|}{ Femur } & Lumbar \\
\hline Normal & 68,56 & 65,18 \\
\hline Osteopenia & 68,25 & 62,49 \\
\hline Osteoporotic & 61,32 & 60,27 \\
\hline
\end{tabular}

There are some limitations in this study. The qualitative evaluation of segmentation needs another method for comparing. As a consequent, actually it could not be proven that the segmented images are robust. On the quantitated evaluation, since the number of normal data is quite large compared to osteopenia and osteoporotic data, and thenthis uneven distribution could lead to accuracy result.More participant are needed to get more data for the future study, and last;more ROI can be considered in an image.

\section{CONCLUSION}

The combination of tophat-bothat filtering, histogram equalization contrasting, and local adaptive thresholding segmentation method can be performed to clearly separate porous trabecular bone in periapical dental X-Ray images. Porous trabecular analysis such as porosity, perimeter of porous, and number of vertex of porous is valuable and promising areas in osteoporosis screening. The experimental resultsshows that the features of porous trabecular bone were used for osteoporosis screening with the classification accuracy around $65 \%$. This result suggests evaluating the segmented image using qualitative method. Improving accuracy rate could be done using different methods.

\section{ACKNOWLEDGMENT}

We thank to DIKTI, The Ministry of Education and Culture of Republic Indonesia for BPPS scholarship, the Prof. Soedomo Dental Hospital, Faculty of Dentistry, Universitas Gadjah Mada and the Dr. Sardjito Hospital, Yogyakarta.for data supporting.

\section{REFERENCES}

[1] M. Park, B. Kang, andS.J. Jin, "Computer aided diagnosis system of medical images using incremental learning method, Intl Journal Expert Systems with Applications", 3, 36, Pergamon Press, Inc. Tarrytown, NY, 2009.

[2] A.A. Tirodkar, "A multi-stage algorithm for enhance X-Ray image segmentation", International Journal of Engineering Science Technology (IJESE), vol. 3, no. 9, September 2011.

[3] F. L.Ding, K. Wee, T. Howe, "Automatic segmentation of femur bones in anterior-posterior pelvis X-Ray images", CAIP 2007: SpringerVerlag, Berlin, 2007.

[4] D. Herumurti, A.Z. Arifin, R. Sulaeman, A. Asano, A. Taguchi, T. Nakamoto, and K. Uchimura, "Weighted fuzzy ARTMAP for osteoporosis detection", Proceeding 16th Korea-Japan Joint Workshop on Frontiers of Computer Vision, Seoul, 2007. 
[5] W.G.M. Geraets, J.G.C. Verheij, P.F. Van der Stelt, K. Horner, C. Lindh, K. Nicopoulou-Karayianni, R. Jacobs, E.J. Marjonovic, J.E. Adams, and H. Devlin, "Selecting regions of interest on intraoral radiographs for predictions of bone mineral density", DentomaxillofacRadiol 37, pp. 375-379, 2008.

[6] B.D. Lee, and S.C. White, "Age and trabecular features of alveolar bone associated with osteoporosis", Oral Surg Oral Pathol Oral RadiolEndod, Elsevier Inc, 2005.

[7] R. Silvakumar, "Identification of early osteoporosis using intensity slicing method", Global Journal of Researches in Engineering General Engineering, vol. 12, issue. 2, version. 1.0, May 2012.

[8] R. Gonzalez, and R.E. Wood, Digital imageprocessing $2^{\text {nd }}$ edition, Prentice-Hall Inc, USA, 2007.

[9] N. Milstein, "Image segmentation by adaptive threshoding", TechnionIsrael Institute of Technology, 1998.

[10] J. Sauvola, and M. PietikaKinen, “Adaptive image binarization”, Pattern Recognition,no. 33, pp. 225-236, 2000
[11] H.J. Sommer, "Area, centroid and area moments for polygonal objects",www.me.psu.edu/ sommer/me481/notes_07_02.doc, accessed $10 \mathrm{Feb} 2013$.

[12] S.K. Pal, and S. Mitra, A review of: neuro-fuzzy pattern recognition methods in soft computing, John Wiley \& Sons, New York, 1999.

[13] A.M. Badawi, "Osteoporosis classification using fuzzy rule based and neural networks", accessed April 2011.

[14] P. Augat and S. Schorlemmer, "The role of cortical bone and its microstructure in bone strength", Age and Ageing, 35-S2: ii27-ii32, Oxford University Press, 2006.

[15] S.C. White, and D.J. Rudolph, "Alterations of the trabecular pattern of the jaws in patients with osteoporosis", Oral Surg Oral Med Oral Pathol Oral Radiol Endod, 88, pp. 628-635, 1999.

[16] Z. Abidin, andA. Z. Arifin, "Analisis kerapatan trabecular bone berbasis graph berbobot pada citra panorama gigi untuk identifikasi osteoporosis", JUTI, vol. 2, no. 7, pp. 59-64, Juli 2009. 